\title{
ESTUDO MORFO-ANATÔMICO DE AMOSTRAS COMERCIAIS DE CATUABA
}

\section{MORPHO-ANATOMIC STUDY OF CATUABA COMERCIAL SAMPLES}

\author{
Flávio Luís Beltrame*; Dalva Cassie Rocha**; \\ Adriana Lenita Meyer Albiero***; Marta Regina Barrotto do Carmo**; \\ Quezia Bezerra Cass****
}

* Professor da Universidade Estadual de Ponta Grossa (UEPG), Departamento de Ciências Farmacêuticas. E-mail: flaviobeltra@gmail.com

** Professora da Universidade Estadual de Ponta Grossa (UEPG), Departamento de Biologia Geral

*** Professora da Universidade Estadual de Maringá (UEM), Departamento de Farmácia

**** Professora da Universidade Federal de São Carlos (UFSCar), Departamento de Química

Recebido para publicação em 18/09/2010

Aceito para publicação em 01/12/2010

\section{RESUMO}

Anemopaegma arvense e Trichilia catigua (catuabas) são usadas por suas ações afrodisíaca e estimulante. Realizou-se estudo microscópico para investigação morfo-anatômica de seis amostras na forma de pó (cápsulas) vendidas em farmácias e de cascas de sete amostras de catuaba comercializadas em feiras populares a fim de subsidiar a identificação da droga vegetal e auxiliar no desenvolvimento de parâmetros farmacognósticos do material seco e pulverizado a ser empregado no controle de qualidade dessas espécies. Desta análise constatou-se que os órgãos subterrâneos de $A$. arvense e as cascas de T. catigua apresentam periderme com poucas camadas de súber e córtex parenquimático, observou-se a presença de idioblastos cristalíferos contendo drusas, xilema e floema secundário. No floema secundário constataram-se anéis de fibras que se separam pelos raios parenquimáticos externamente, há fileiras de cristais prismáticos nas células que acompanham as fibras tanto no xilema quanto no floema na amostra de T. catigua. Os resultados demonstraram que as amostras comercializadas na forma de droga vegetal apresentaram características semelhantes às observadas para o padrão de T. catigua e amostras comercializadas como cápsulas do pó. Além disso, uma amostra apresentou características distintas das demais e dos padrões avaliados. A microscopia mostrou-se uma ferramenta eficiente na identificação das amostras comercializadas e provou ser uma técnica adequada para a avaliação deste material em complementação/substituição a técnicas mais avançadas de análise.

Palavras-chave: Anemopaegma arvense. Controle de qualidade. Microscopia. Trichilia catigua. 


\begin{abstract}
Anemopaegma arvense and Trichilia catigua (catuaba) are used for their aphrodisiac and sexual stimulant actions. Microscopic study was undertaken to investigate the morphology and anatomy of six samples - in powder form (capsules) - , sold in pharmacies, and seven catuaba's barks sold in street markets in order to identify the drug and help the development of pharmacognostic parameters of dry and pulverized material to be used in quality control of these species. It was observed that $A$. arvense material and the bark of $T$. catigua periderm have few layers of suber and parenchymatic cortex; it was also observed the presence of idioblasts with druses; secondary xylem and phloem. It was observed ring fibers that are separate by parenchyma rays externally; there are rows of prismatic crystals in cells that follow the fibers in the xylem and phloem in the sample of T. catigua. The results showed that the samples sold in the form of dried plant (drug) and samples sold as capsules (powder form) have similar characteristics to the pattern observed for T. catigua. In addition, one of the samples showed distinct characteristics from other samples and patterns evaluated. Microscopy proved to be an effective tool in the identification of the samples and proved to be an adequate technique for the evaluation of this material to complement/replace the more advanced techniques of analysis.
\end{abstract}

Keywords: Anemopaegma arvense. Microscopy. Quality control. Trichilia catigua.

\section{Introdução}

Atualmente, de forma cada vez mais acentuada, o mercado farmacêutico vem procurando alternativas terapêuticas para o tratamento de problemas relacionados com a disfunção erétil e a falta de libido, principalmente produtos originários de plantas com ação afrodisíaca ou capacidade de aumentar o prazer sexual. Neste sentido, é comum encontrar em farmácias e estabelecimentos que comercializam plantas medicinais, e até mesmo na internet, a venda de produtos que se propõem a atender a essas expectativas, muitas vezes prometendo os efeitos desejados sem efeitos colaterais (VEIGA, PINTO, 2005).

Um dos produtos consumidos no Brasil para este fim é o órgão subterrâneo de uma planta comercializada in natura conhecido popularmente como catuaba (MARQUES, 1998). É usada na forma de fragmentos secos para infusões ou imersa em veículo alcóolico ou ainda comercializada na forma de pó.

No entanto, em diferentes regiões do Brasil, diferentes vegetais são conhecidas com o mesmo nome, tais como Anemopaegma sp. (Bignoniaceae), Erythroxylum sp. (Erythroxylaceae), Illex sp. (Aquifoliaceae), Micropholis sp. (Sapotaceae),
Secondatia sp. (Apocynaceae), Tetragastris sp. (Bursereceae) e Trichilia sp. (Meliaceae) (DUCKE, 1966; PEREIRA, 1982; JORGE, et al., 1989; MARQUES, 1998; SATOH, et al., 2000; ZANOLARI, et al., 2003; KLETTER, et al., 2004; SILVA, 2004; UCHINO, et al., 2004).

A espécie vegetal registrada como catuaba para fins medicinais na Farmacopéia Brasileira é a Anemopaegma arvense (Veil.) Stellfeld (Bignoniaceae) (FARMACOPÉIA DOS ESTADOS UNIDOS DO BRASIL, 1929). Por outro lado, a espécie vegetal mais comumente comercializada como catuaba no mercado farmacêutico brasileiro é Trichillia catigua A. Juss (Meliaceae) (BELTRAME, et al., 2005), sendo a casca empregada para diversas finalidades medicinais (KLETTER, et al., 2004).

Esta realidade proporciona grandes dificuldades na identificação da matéria prima vegetal adquirida e empregada para o preparo de medicamentos. Além disto, a avaliação da qualidade destes produtos e a determinação de suas características é, em muitos casos, inexistente.

Embora o comércio brasileiro tenha apresentado um aumento do número de fitoterápicos disponíveis à população e, conseqüentemente do consumo, este incremento no uso não foi acom- 
panhado pelo desenvolvimento e aprimoramento de técnicas para a determinação das características de qualidade dos produtos e também da fiscalização do setor produtivo. A ocorrência de fraudes e a má qualidade de fitoterápicos comercializados têm preocupado profissionais da área de saúde e a comunidade científica. Os desvios de qualidade e a adulteração interferem na eficácia e até mesmo na segurança para sua utilização (CHIMIN et al., 2008)

Diversos autores têm demonstrado em seus artigos, grandes distorções entre a qualidade dos produtos analisados e os parâmetros estabelecidos pela legislação sanitária confirmando desvios de qualidade em produtos, inclusive industrializados (OLIVEIRA, AKISUE, 1989; NASCIMENTO, et al, 2005; CHIMIN, et al., 2008; BELTRAME, et al., 2009).

Visando contribuir para melhor qualidade dos medicamentos fitoterápicos, a Cromatografia Líquida de Alta Eficiência (CLAE) tem sido empregada pelas grandes indústrias farmacêuticas, nacional e estrangeira, na avaliação das características de qualidade das matérias primas vegetais usadas no preparo de diversos medicamentos.

Por outro lado, para pequenas indústrias e farmácias de manipulação, o custo de análise por essa técnica torna-se impraticável, sendo que o emprego de métodos como a análise microscópica (OLIVEIRA, AKISUE, 1989) - recomendada pela Farmacopéia Brasileira - é uma alternativa viável e aceita pelos órgãos de inspeção e controle sanitário do Brasil (BRASIL, 2010).

Sendo assim, o estudo aqui apresentado tem como escopo avaliar morfologicamente diferentes preparações comerciais de catuaba e analisar comparativamente com padrões de $A$. arvense e T. catigua colaborando com o estabelecimento de parâmetros para o controle de qualidade.

\section{Material e Métodos}

\section{Material vegetal padrão}

Cascas de T. catigua foram coletadas na cidade de Maringá (Paraná, Brasil) no mês de outubro de 2001, no Horto Florestal Dr. Luis Teixeira
Mendes. A planta foi identificada e uma exsicata foi depositada no Herbário da Universidade Estadual de Maringá com o número HUEM-9908. Órgãos subterrâneos de $A$. arvense foram coletados na cidade de Itirapina (São Paulo, Brasil) no mês de novembro de 2001, no Parque Estadual de Itirapina e uma exsicata foi depositada no Herbário da Universidade Estadual Paulista de Rio Claro sob o número HRCB-3542, para fins de identificação.

\section{Amostras comercias de Catuaba}

Amostras comerciais de catuaba foram adquiridas em feiras livres e farmácias em diferentes Estados do território brasileiro, sendo 6 (seis) amostras moídas e encapsuladas (três do Estado do Paraná e três de São Paulo) e 7 (sete) amostras dessecadas e fragmentadas, aqui denominadas cascas, provenientes do Pará, Maranhão, Rio Grande do Norte, Rio Grande do Sul, Pernambuco, Distrito Federal e Ceará.

\section{Caracterização morfo-anatômica}

Para a caracterização morfo-anatômica das amostras padrão, foi realizada a fixação em FAA 50 e posterior conservação em etanol $70 \%$ (JOHANSEN, 1940). A análise microscópica destas amostras foi realizada segundo as recomendações usuais da farmacognosia a partir de secções anatômicas à mão livre, com auxílio de lâminas de aço, das amostras conservadas em etanol 70\% (OLIVEIRA, AKISUE, 1989). O material foi descorado com hipoclorito de sódio (30\% a partir de solução comercial), lavadas e coradas com azul de astra e safranina a 1\% (safrablau) e lâminas semi-permanentes foram montadas em gelatina glicerinada (KRAUS, ARDUIN, 1997).

Para a caracterização das amostras comercializadas em cápsulas, os padrões de $T$. catigua e $A$. arvense foram estabilizados em estufa de ar circulante a temperatura de $40^{\circ} \mathrm{C}$, triturados e tamizados manualmente, em malha de 200 mesh $(75 \mu \mathrm{M})$ (BRITO, 2004). Os pós das amostras comerciais e dos padrões foram diafanizados com solução de hipoclorito de sódio a $30 \%$ durante 15 minutos, lavados com água destilada e corados e foram montadas laminas semi-permanentes, conforme descrito anteriormente. 
As cascas foram tratadas com uma técnica de maceração, comum para a análise de células isoladas, após a dissociação da lamela média a partir da ação de peróxido de hidrogênio e ácido acético, pelo método de Franklin modificado (KRAUS, ARDUIN, 1997).

Os aspectos anatômicos foram observados, descritos e registrados por meio de fotomicrografias obtidas em microscópio Olympus ${ }^{\circledR}$ e as imagens capturadas pelo programa Image Pró-plus ${ }^{\circledR}$ versão 4.0. As escalas referentes às ilustrações foram obtidas utilizando-se lâminas micrométricas nas mesmas condições ópticas utilizadas em cada caso.

\section{Resultados}

Neste estudo, as características observadas nas seções transversais e longitudinais do órgão subterrâneo de $A$. arvense (Figuras 1A-B) e da casca de $T$. catigua (Figuras 1C-E) e no material pulverizado destes, foram consideradas como padrões para a análise farmacobotânica.

Tanto o órgão subterrâneo de $A$. arvense como a casca do caule de $T$. catigua anatomicamente apresenta estrutura caulinar secundária completa caracterizada pela periderme, floema e xilema secundários (Figura 1A-D).

Em seção transversal, nota-se que $A$. arvense possui o córtex mais estreito que $T$. catigua cujo córtex parenquimático tem cerca de vinte camadas de células, entre as quais ocorrem numerosos idioblastos cristalíferos, contendo drusas, mais concentrados nas proximidades do súber. A periderme possui poucas camadas de súber nas duas espécies. $\mathrm{O}$ xilema secundário tem distribuição difusa uniforme dos elementos de vaso em ambos os materiais. Há elementos de vasos múltiplos (de dois) em $A$. arvense e predominantemente solitários em $T$. catigua. $\mathrm{O}$ agrupamento de fibras no floema secundário difere nos dois materiais, pois formam pequenos grupos em $A$. arvense e formam anéis em $T$. catigua, sobretudo quando estão mais próximas do xilema nesta espécie vegetal. Largos raios parenquimáticos são notáveis próximos a região do câmbio vascular em $A$. arvense, o que é pouco visível em $T$. catigua.
Dois aspectos a destacar que foram observados apenas em de T. catigua: i) drusas no córtex (Figura 1D); ii) fileiras de cristais prismáticos presentes nas células que acompanham as fibras tanto no xilema quanto no floema, visíveis em seções longitudinais da estrutura caulinar (Figura 1E).

Foi possível observar ainda, a presença de grãos de amido em todas as células parenquimáticas em $A$. arvense (não ilustrado), indicando outra característica para distinção com $T$. catigua.

Figura 1 - Padrões de catuaba. A-B Anemopaegma arvense, seção transversal do órgão subterrâneo de. C-E Trichilia catigua, seção transversal (CD) e longitudinal (E) do caule. Destacam-se cristais prismáticos (cp), cambio vascular (cv), elementos de vasos (ev), fibras de floema (ff), floema secundário (fs), periderme (pr), raios parenquimáticos (rp), drusas ( ).
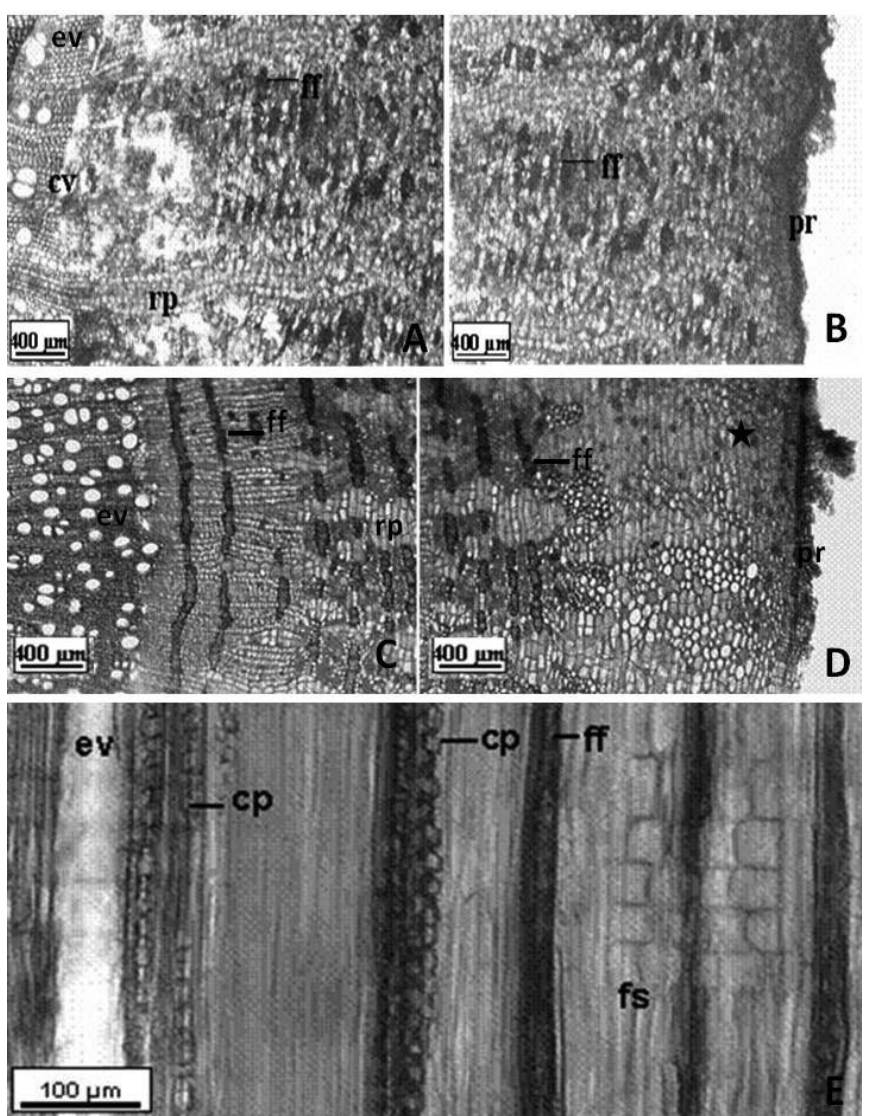

A Figura 2 apresenta fragmentos do material comercializado na forma de pó encapsulado, obtido em diversas farmácias dos Estados do Paraná e São 
Paulo. Os dados da comparação dos padrões com as sete amostras de catuaba na forma de pó encapsulado comercializadas estão mostradas na Tabela 1 .

Figura 2 - Amostras catuaba moída e encapsulada, obtidas no comércio - Fragmentos de caule seco e triturado evidenciando cristais prismáticos (cp) em fibras de xilema secundário (fx) e fragmentos de parênquima (pa).

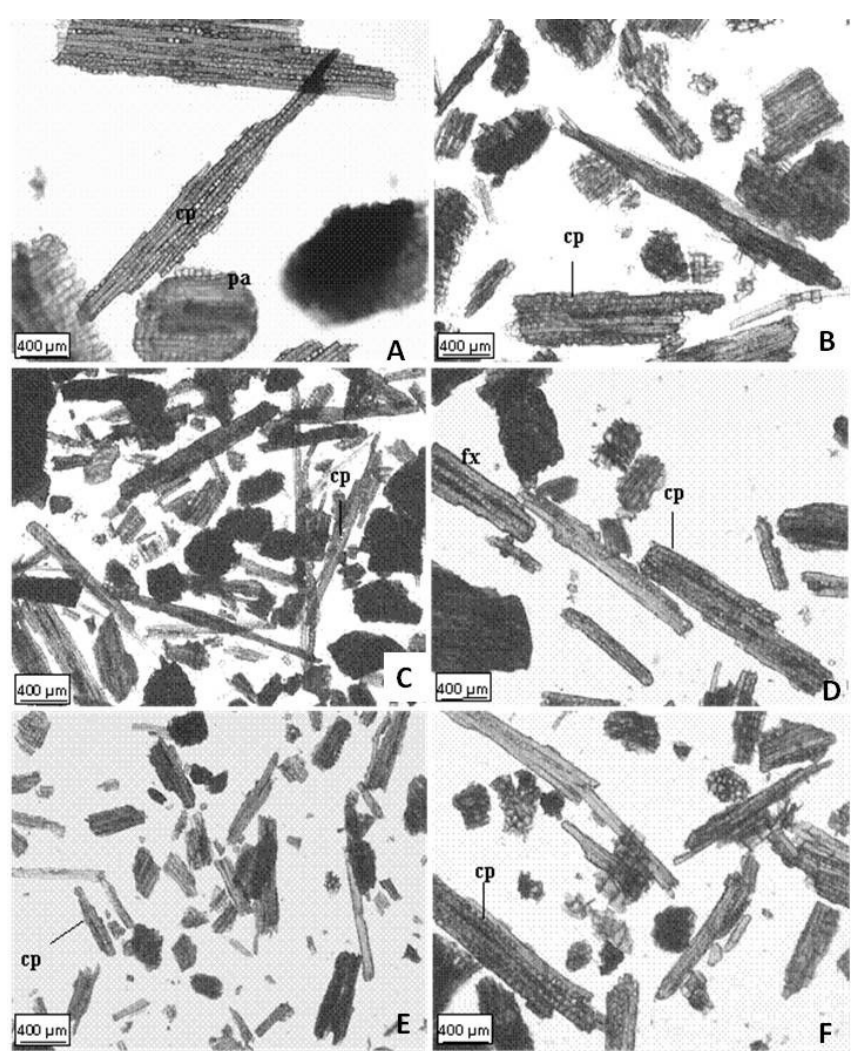

A Figura 3 corresponde às amostras comercializadas que foram adquiridas na forma de casca e cuja análise farmacobotânica foi precedida de maceração.

A análise das amostras fragmentadas revelou que em todas havia células de parênquima e de epiderme e ainda fibras contendo cristais e alguns poucos elementos de vaso (Figuras 3 A-G). Nas amostras do Pará e do Maranhão (Figuras $3^{\mathrm{a}}$ e B) foram observados mais cristais isolados junto ao parênquima que cristais posicionados em fileiras no interior de fibras. Ainda, nas amostras do Rio Grande do Sul e do Distrito federal (Figuras 3D e F), foram observadas muitas fileiras de cristais prismáticos, algumas células de epiderme e de parênquima, e poucos elementos de vaso. Por fim, nas amostras do Pará, Rio Grande do Norte e Distrito Federal (Figuras 3B, C e F) foram observadas muitas aglomerações de células epidérmicas.

Figura 3 - Amostras de catuaba comercializadas in natura, como fragmentos de casca.
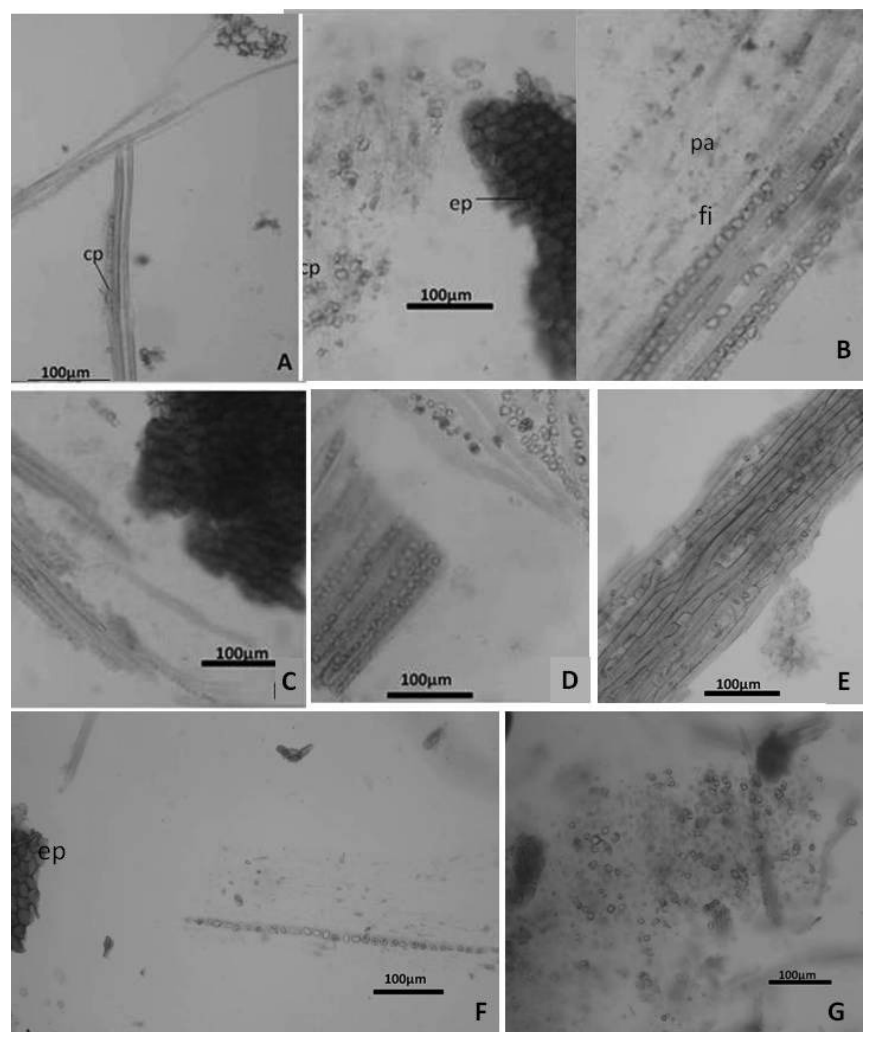
Tabela 1 - Amostras de "Catuaba": Características diagnósticas observadas entre os padrões de $A$. arvense e T. catigua, e em amostras (material moído e encapsulado) comercializados em farmácias de manipulação.

\begin{tabular}{|c|c|c|c|c|c|c|c|c|}
\hline Amostras & Padrão & Padrão & Amostra & Amostra & Amostra & Amostra & Amostra & Amostra \\
\hline Caracteres & $\mathrm{Aa}$ & $\mathrm{Tc}$ & 1-PR & 2-PR & 3-PR & 4-SP & 5-SP & 6-SP \\
\hline Coloração & Am & Av & Av & Av & Av & Av & Av & Av \\
\hline $\begin{array}{l}\text { Fileiras de cristais no } \\
\text { floema }\end{array}$ & - & $\mathbf{X}$ & $\mathbf{X}$ & $\mathbf{X}$ & $\mathbf{X}$ & $\mathbf{X}$ & $\mathbf{X}$ & $\mathbf{X}$ \\
\hline $\begin{array}{l}\text { Fileiras de cristais no } \\
\text { xilema }\end{array}$ & - & $\mathbf{X}$ & $\mathbf{X}$ & $\mathbf{X}$ & $\mathbf{X}$ & $\mathbf{X}$ & $\mathbf{X}$ & $\mathbf{X}$ \\
\hline $\begin{array}{l}\text { Células com grãos de } \\
\text { amido }\end{array}$ & $\mathbf{X}$ & - & - & - & - & $\mathbf{X}$ & - & - \\
\hline $\begin{array}{l}\text { Drusas e cristais prismáti- } \\
\text { cos isolados }\end{array}$ & - & $\mathbf{X}$ & $\mathbf{X}$ & $\mathbf{X}$ & $\mathbf{X}$ & $\mathbf{X}$ & $\mathbf{X}$ & $\mathbf{X}$ \\
\hline Impurezas (areia) & - & - & - & - & - & $\mathbf{X}$ & - & - \\
\hline
\end{tabular}

Aa. A. arvense. Tc. T. catigua. Am. Amarelo pardo. Av. avermelhado. - não observado. X. observado, SP: São Paulo, PR: Paraná.

\section{Discussão}

O trabalho de comparação das características estruturais entre as amostras é considerado uma diagnose ou análise farmacobotânica (OLIVEIRA, AKISUE, 1989). Neste trabalho, as amostras comerciais foram comparadas com as amostras identificadas em Herbários.

A análise microscópica das amostras comercializadas em pó revelou que as cápsulas de catuaba apresentam material vegetal moído em diferentes padrões granulométricos.

Foi possível observar principalmente, que a amostra 4, apesar de apresentar características semelhantes ao padrão de T. catigua, apresentava grãos de amido e areia. A presença de grãos de amido pode sugerir uma adição fraudulenta deste material ou mesmo a contaminação da amostra com partes da espécie vegetal $A$. arvense. Entretanto, não se pode concluir se há presença desta ou de outro vegetal.
As demais amostras em pó apresentaram várias semelhanças estruturais com as características da espécie padrão T. catigua.

A presença de elementos de vaso, célula característica do xilema, indica que, às vezes os fragmentos das "cascas" podem conter tecidos mais internos e não apenas a periderme (COSTA et al.,2003). Foi possível também verificar que as fileiras de cristais se formam porque estas substâncias inorgânicas se encontram no interior de fibras septadas cujas paredes possuem muitas pontuações. Essas células também são componentes associados tanto no floema como no xilema. Na literatura, a presença de uma bainha de fibras septadas próximas ao floema em pulvino de leguminosas, indica seu papel no transporte simplástico de substâncias devido às numerosas pontuações (RODRIGUES, MACHADO, 2006). A presença de cristais no interior dessas células pode reforçar este conceito, uma vez que o protoplasto é mantido. 
Quanto à presença de cristais de oxalato de cálcio nas amostras, muitas vezes, isto é um indicativo de toxicidade da planta, no entanto, já se discute que essa toxidade pode estar relacionada à associação dessas substâncias com outros produtos orgânicos como lipídios e, principalmente, proteínas (FERREIRA, MARSOLA, TEIXEIRA, 2011). No caso de catuaba, essas fileiras de cristais de oxalato de cálcio já foram descritas como característica de T. catigua por outros autores (LAGO, MIGUEL, DUARTE, 2007), enquanto que nada foi mencionado sobre essas substâncias para A. arvense (MAURO, et al., 2007).

\section{Conclusão}

Do ponto de vista morfo-anatômico, com exceção da amostra 4 , as cascas, assim como as cápsulas de planta moída, todas comercializadas como catuaba, apresentaram características peculiares à amostra padrão de T. catigua.

Assim sendo, a microscopia mostrou-se uma ferramenta eficiente na identificação e avaliação das amostras comercializadas dessecadas e fragmentadas e provou ser uma técnica adequada para a avaliação destas amostras em substituição a técnicas mais avançadas de análise.

Além disso, permitiu comprovar que se trata de uma ferramenta eficaz para a determinação da presença de impurezas e contaminantes em amostras.

Por fim, a técnica ainda apresenta-se menos onerosa em relação às mais avançadas a serem aplicadas no controle de qualidade de fitoterápicos, sendo indicadas como procedimento a ser usado inclusive por estabelecimentos farmacêuticos de pequeno porte.

Agradecimentos: Ao CNPq pelo apoio financeiro; ao PIBIC/CNPq/UEPG pela concessão de bolsas de iniciação científica e a Farmacêutica Daiana Ferroni pela realização dos cortes histológicos.

\section{REFERÊNCIAS}

BELTRAME, F.L., et al. A validatedhigher-performance liquid chromatography method for quantification of cinchonain Ib inbark and phytopharmaceuticals of Trichilia catigua used as catuaba. Jounal of Chromatography A, Nova Iorque, v.1119, p. $257-263,2005$.

et al. Avaliação da qualidade das amostras comercias de Baccharis trimera L. (Carqueja) vendidas no Estado do Paraná. Acta Scientiarum. Health Sciences, Maringá, v. 31, n. 1, p. 37-43, 2009.

BRASIL. Ministério da Saúde. Agência Nacional de Vigilância Sanitária. Resolução de Diretoria Colegiada (RDC) n 14 , de 05 de abril de 2010. Dispõe sobre o registro de medicamento fitoterápico. Diário Oficial da República Federativa do Brasil, Brasília, 05 abril 2010.

BRITO, M. P. Estudos farmacognósticos de Smallanthus sonchifolius (Asteraceae) e obtenção de seus extratos flúido e seco. Ribeirão Preto, 2004. Dissertação de Mestrado, Faculdade de Ciências Farmacêuticas de Ribeirão Preto, Universidade de São Paulo.

CHIMIN, A. et al. Avaliação da qualidade de amostras comerciais de Maytenus ilicifolia (espinheira-santa) comercializadas no Estado do Paraná. Latin America Journal of Pharmacy, Buenos Aires, v. 27, n. 4, p. 591-597, 2008.

COSTA, C. G. et al. Xilema. In: GLÓRIA, B. A.; Guerreiro, S. M. C.(Eds.). Anatomia vegetal. Viçosa: UFV, 2003.

DUCKE, A. A Catuaba na botânica sistemática, científica e pseudo-científica. Revista Brasileira de Farmácia, Rio de Janeiro, v. 5, set-out, p.3-8, 1966.

FARMACOPÉIA DOS ESTADOS UNIDOS DO BRASIL. São Paulo, Companhia Editora Nacional, 1929, p. 194.

FERREIRA, L. S.; MARSOLA, F. J.; TEIXEIRA, S. P. Anatomia dos órgãos vegetativos de Dieffenbachia picta Schott (Araceae) com ênfase na distribuição de cristais, laticíferos e grãos de amido. Revista Brasileira Farmacognosia, v.16, suppl. p. 664-670, 2011.

JOHANSEN, D. A. Plant microtechnique. New York: McGraw-Hill, 1940. 523p.

JORGE, L. I. F. et al. Determinação das principais características estruturais e químicas da droga Anemopaegma (VELL) STELLFELD (CATUABA). Revista Instituto Adolfo Lutz, Rio de Janeiro, v. 49, n. 2, p. 183-191, 1989.

KLETTER, C., et al. Morphological, chemical, and functional analysis of Catuaba preparations. Planta Médica, Nova Iorque, v. 70, p. 993-1000, 2004.

KRAUS J.; ARDUIN M. Manual básico de métodos em morfologia vegetal. Seropédica: EDUR, 1997. 198p. 
LAGO, J. B.; MIGUEL, O. G.; DUARTE, M. R. Caracteres anatômicos de catuaba (Trichilia catigua A. Juss., Meliaceae). Latin American Journal Pharmacy, v.26, n. 2, p. 185-90, 2007.

MARQUES, L. C. Contribuição ao Esclarecimento da Identidade Botânica da droga Vegetal Catuaba. Revista Racine, São Paulo, v. 3, n. 43, p. 8-11, 1998.

MAURO, C., et al.. Estudo anatômico das espécies de cerrado Anemopaegma arvense (Vell.) Stellf. ex de Souza (catuaba), Zeyheria montana Mart. (bolsa-de-pastor) e Jacaranda decurrens Chamisso (caroba) - Bignoniaceae. Revista Brasileira Farmacognosia, v.17, n.2, p. 262-265, 2007.

NASCIMENTO, V. T., et al. Controle de qualidade de produtos à base de plantas medicinais comercializados na cidade do Recife-PE: erva-doce (Pimpinella anisum L.), quebra-pedra (Phyllanthus spp.), espinheira santa (Maytenus ilicifolia Mart.) e camomila (Matricaria recutita L.). Revista Brasileira de Plantas Medicinais, Botucatu, v. 7, n. 3, p. 56-64, 2005.

OLIVEIRA, F.; AKISUE, G. Fundamentos de farmacobotânica. São Paulo: Atheneu, 1989. 216 p.

PEREIRA, N. A. Contribuição de Manuel Freire Alemão de Cisneiros para o conhecimentos de nossos fitoterápicos. Rio de Janeiro: Brasileira de Artes Gráficas, 1982. 600 p.

RODRIGUES, T. M.; MACHADO S. R. Anatomia comparada do pulvino primário de leguminosas com diferentes velocidades de movimento foliar. Revista Brasileira Botânica, v.29, n.4, p.709-720, 2006.

SATOH, M., et al. Cytotoxic constituents from Erytroxylum catuaba isolation and cytotoxic activities of cinchonain. Natural Medicines, v. 54, n. 2, p. 97-100, 2000.

SILVA, A. J. Estudo Botânico e chímico da Catuaba (Erythroxylaceae catuaba do Norte). Revista Brasileira de Farmacognosia, Curitiba, v. 14, n.1, p. 67-77, 2004.

UCHINO, T., et al. Potent protecting effects of Catuaba (Anemopaegma arvense) extracts against hydroperoxideinduced cytotoxicity. Toxicology in vitro, Amsterdã, v. 18, p. $255-263,2004$.

VEIGA, V. F. J.; PINTO, A. C. Plantas medicinais: cura segura? Quimica Nova, São Paulo, v. 28, n. 3, p. 519-528, 2005.

ZANOLARI, B., et al. On-line identification of tropane alkaloids from Erytroxylum vacciniifolium by liquid chromatography-UV detection-multiple mass espectrometry and liquid chromatography -nuclear magnetic ressonance spectrometry. Journal of Chromatography A, Nova Iorque, v. 1020, p. 75-89, 2003. 